\title{
Active Elbow Joint Model
}

\author{
Hyung Yun Choi ${ }^{1} \quad$ Manyong Han ${ }^{1}$ Whe-Ro Lee ${ }^{1}$ \\ Toru Matsui $^{2}$ Hisayoshi Matsuoka ${ }^{2}$ \\ ${ }^{1}$ Mechanical System Design Engineering Dept., Hongik University, Korea, hychoi@hongik.ac. kr \\ ${ }^{2}$ Integrated CAE and PLM Dept., Nissan Motor Co, Ltd., Japan
}

\begin{abstract}
Voluntary and reflexive muscle activation of human elbow joint is investigated by both subject tests and numerical simulations. A jerk loading is applied to extend the elbow joint with different muscle tensing and pre-recognition conditions. Inter- and Intra-subject variations of hand displacement are analyzed for an objective assessment of the active response at the elbow joint to the external perturbation. Both Modelica and finite element mesh models are developed using passive kinematic joint element and active torque which has PID close loop control. The simulation result from these two models are compared with test results and shows a good correlation.
\end{abstract}

Keywords: Digital human body model, Voluntary and reflexive muscle activation

\section{Introduction}

Digital human body models (DHBM) have been widely adopted in various $\mathrm{CAE}$ processes of vehicle design, e.g., car crash simulation for the prediction of injury risk and riding comfort simulation for the assessment of occupant discomfort. For most of such cases, the DHBM is in 3D finite element mesh shape so that it can mechanically interact with vehicle structures such as seat, safety belt and airbag. Thanks to efforts from many researchers, there is a significant advancement in human body modeling (www.ghbmc.com), e.g., mechanical behavior of biological tissue but the active human response with voluntary and reflexive muscle activation that affects occupant kinematics are still remaining as a great challenge.

Muscle tensing of bracing occupant produces larger axial forces, stress redistribution within bones, increase in effective mass and stiffness, altered kinematics, and less excursion and smaller joint rotations (Choi, 2005). Voluntary and reflexive muscle activation of a vehicle occupant is modeled by active joint element at each anatomical joint position (e.g., shoulder, knee, spine, and etc.). There are two basic elements at each joint, i.e., passive kinematic joint elements and torque actuators. Assuming that a co-contraction of agonist and antagonist muscles stiffens the joint articulation, spring constant and damping coefficient of the passive kinematic joint element are adjusted for the different level of co-contraction, which is considered as a major mechanism of voluntary muscle activation. A vestibular reflexive muscle activation for the posture stabilization is modeled by active torque with PID close loop control. Active torque, the control signal is a sum of proportional, integral, and derivative terms between current and reference states of the joint angle.

Test of jerk loading applied to elbow joint which is relatively simple one dimensional articulation is performed with live human subjects to identify and quantify the active response with different muscle conditions. Two kinds of numerical elbow models, i.e., 3D finite element mesh and Modelica models are built to reproduce the active response to the jerk loading and further to elucidate those kinesiologic behavior of bracing human joint.

\section{Jerk loading to elbow joint extension}

During the vehicle driving or just riding, external loadings are often applied to the occupant as perturbations, e.g., vertical bumping on rough road, lateral $\mathrm{G}$ force at cornering, and autonomous braking with ADAS (Advanced Driver Assistant System). It would be quite natural that the occupant spontaneously brace to keep his (or her) upright sitting posture. In order to mimic this kind of perturbation of vehicle in motion and bracing behavior of the occupant, jerk loadings to elbow joint extension are performed as follows.

\subsection{Anthropometry of test subjects}

Five male subjects are recruited and their average age and anthropometric data are listed in Table 1.

Table 1 Average data (SD) of five test subjects

\begin{tabular}{|c|c|c|c|c|}
\hline age & height & $\begin{array}{c}\text { Weight } \\
(\mathrm{kg})\end{array}$ & $\begin{array}{c}\text { Fat Free } \\
\text { Mass }(\mathrm{kg})^{*}\end{array}$ & $\begin{array}{c}\text { forearm } \\
\text { weight }(\mathrm{kg})^{* *}\end{array}$ \\
\hline 28 & 172 & 72.8 & 53.9 & 1.714 \\
$(2.3)$ & $(4.6)$ & $(5.1)$ & $(2.1)$ & $(0.11)$ \\
\hline
\end{tabular}

*: from inbody analysis

**: calculated from GEBOD (Huaining Cheng, 1996) 


\subsection{Jerk loading test}

The elbow joint with simple 1-DOF is selected. Upper body and upper arm of test subject are restrained and the elbow joint angle is to maintain its initial position, i.e., keeping the forearm levelled before and after the jerk loading. There are two kind of loadings, $5 \mathrm{kgf}$ static loading on hand and $3 \mathrm{kgf}$ jerk loading on wrist which is initially carried by a string and just becomes a jerk load when string is cut. (See Fig. 1). The subject has two test conditions, 1) co-contraction versus single contraction and 2) recognition versus unrecognition of jerk loading. Co-contraction or single contraction is respectively attempted by contracting both agonist (e.g., biceps) and antagonist (e.g., triceps) muscles or only agonist muscles. Recognition of the jerk loading to test subject is made by letting him to make his own observation of the action of string cut, i.e., open eye condition. On the contrary, the closed eye condition does not allow the test subject to become aware of the precise moment string cut. There are thus a total four cases of test conditions, "open eye tensed" (recognized with co-contraction), "closed eye tensed" (unrecognized with co-contraction), "open eye relaxed" (recognized with single contraction), and "closed eye relaxed" (unrecognized with single contraction). All five test subjects have two trials for each case of four test conditions.

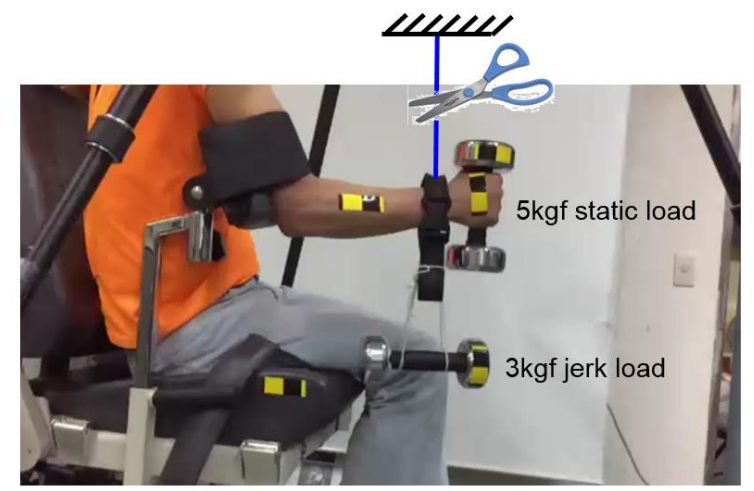

Figure 1 Test setup for jerk loading at elbow joint

\subsection{Measurement of hand motion}

Hand displacements, digitized from video, are shown in Fig. 2. Intra-subject variations are quantitatively assessed by CORA (CORrelation and Analysis, http://www.pdb-org.com/de/information/18-coradownload.html) score as listed in Table 2. All five test subjects showed high CORA scores with "open eye relaxed" condition, i.e., good repeatability between two trials at recognized with single contraction muscle condition. It is speculated that the cases with low CORA score are due to the poor coordination of muscle tensing condition of the subject, e.g., closed eye relaxed case with test subject \#1.

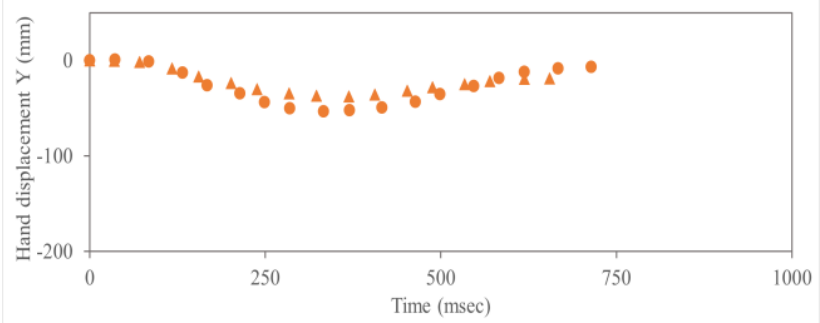

(a) Open Eye Tensed

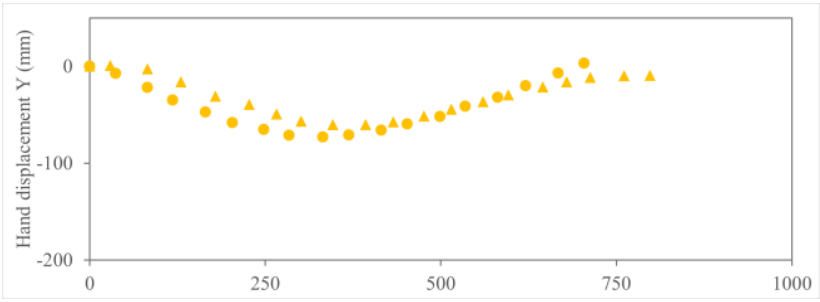

(b) Close Eye Tensed

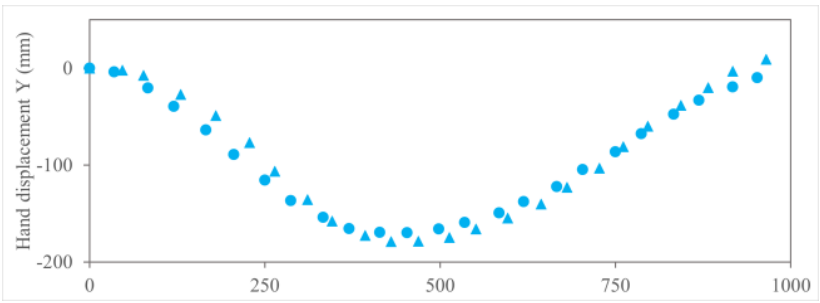

(c) Open Eye Relaxed

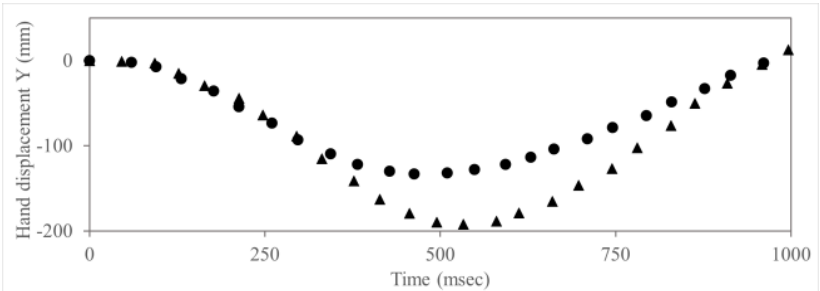

(d) Close Eye Relaxed

Figure 2 Typical hand displacements in vertical direction (y) due to the jerk loading from subject \#2 (•: $\left.1^{\text {st }} \operatorname{try}, \mathbf{\Delta}: 2^{\text {nd }} \operatorname{try}\right)$

Table 2 CORA score for intra-subjects variation

\begin{tabular}{|c|c|c|c|c|}
\hline $\begin{array}{c}\text { Sub. } \\
\#\end{array}$ & $\begin{array}{c}\text { Open eye } \\
\text { Tensed }\end{array}$ & $\begin{array}{c}\text { Close eye } \\
\text { Tensed }\end{array}$ & $\begin{array}{c}\text { Open eye } \\
\text { Relaxed }\end{array}$ & $\begin{array}{c}\text { Close eye } \\
\text { Relaxed }\end{array}$ \\
\hline 1 & 0.699 & 0.967 & 0.957 & 0.493 \\
\hline 2 & 0.746 & 0.732 & 0.948 & 0.768 \\
\hline 3 & 0.962 & 0.642 & 0.955 & 0.898 \\
\hline 4 & 0.365 & 0.828 & 0.720 & 0.851 \\
\hline 5 & 0.546 & 0.766 & 0.795 & 0.914 \\
\hline Mean & 0.693 & 0.787 & 0.875 & 0.785 \\
(S.D.) & $(0.21)$ & $(0.11)$ & $(0.10)$ & $(0.15)$ \\
\hline
\end{tabular}

The inter-subject variation is also represented by test corridors with mean hand displacements as shown in Fig. 3. The open eye tensed condition shows the least width between upper and lower corridors while the open eye relaxed condition has largest. 


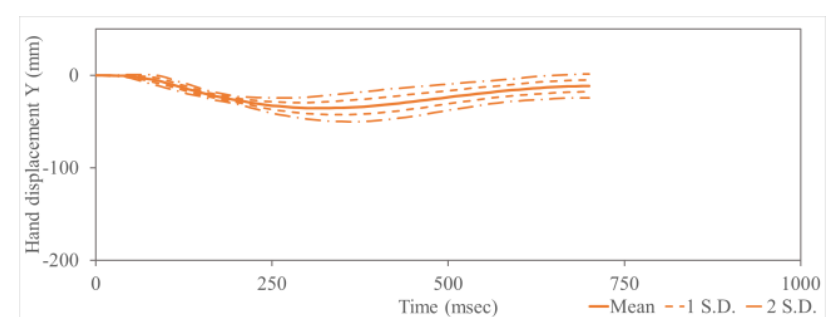

(a) Open Eye Tensed

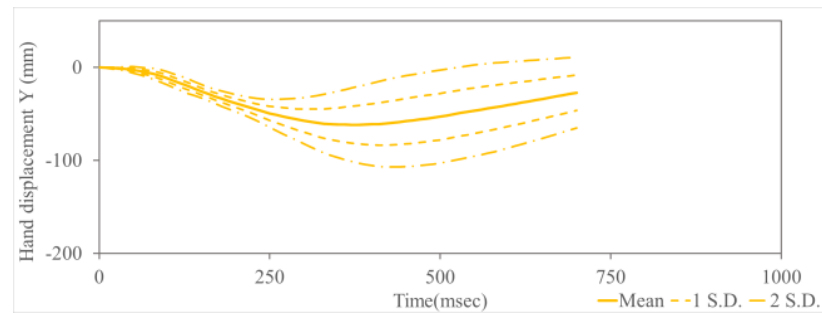

(b) Close Eye Tensed

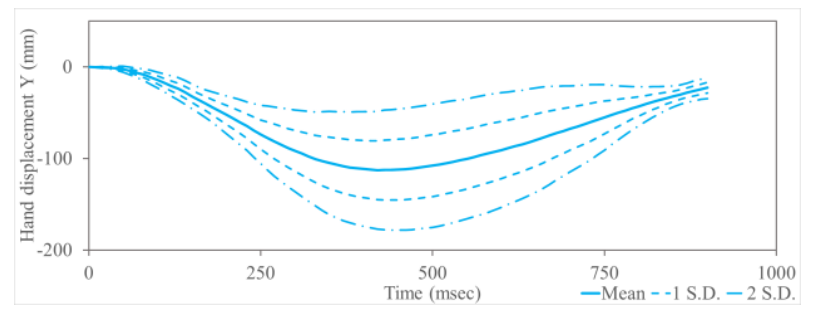

(c) Open Eye Relaxed

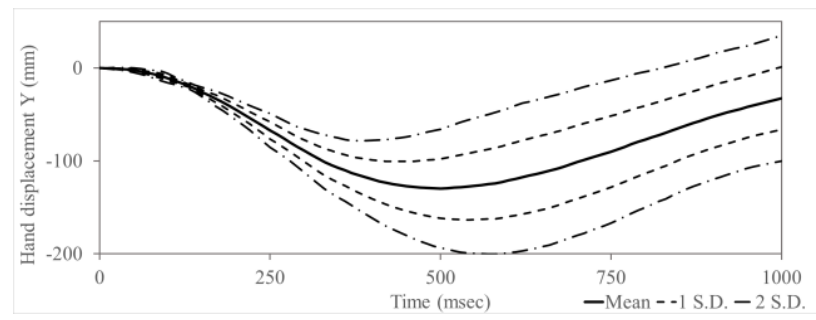

(d) Close Eye Relaxed

Figure 3 Test corridors and mean hand displacements of three subjects.

\section{Three dimensional finite element mesh versus Modelica models}

Both FE mesh and Modelica models are shown in Fig. 4. Two rigid bodies, i.e., upper and lower arms articulated by one dimensional kinematic joint element that represents the elbow joint. The dynamic properties of two rigid body are assigned from the average data of five test subjects. It is confirmed that the outcome of both model, i.e., elbow extension and hand displacement from the jerk loading is identical to each other.

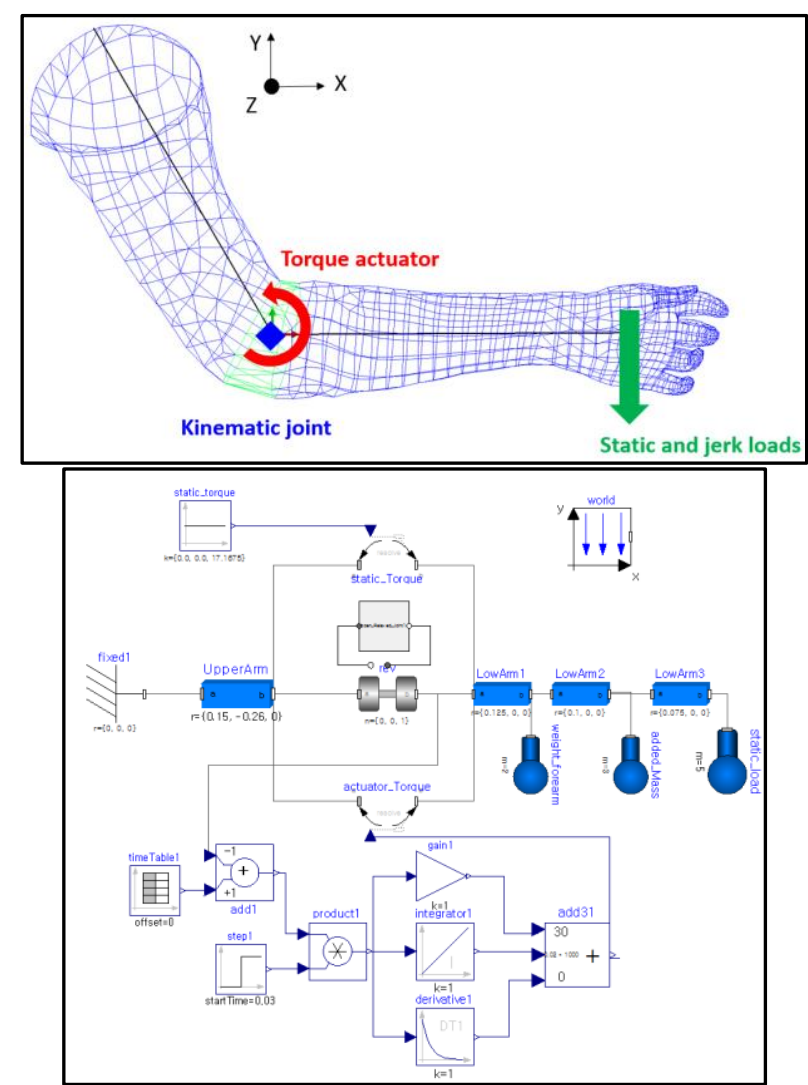

Figure 4 3D finite element mesh (top) and Modelica (bottom) models of active elbow joint

\subsection{Modeling of active elbow joint}

The numerical modeling of elbow joint and its active response to the jerk loading is designed by implementing two mechanical components, a passive 1D kinematic joint element and a torque actuator. The linear stiffness and damping coefficient of the passive 1D kinematic joint element present the level of cocontraction that stiffens the elbow joint articulation. Voluntary and reflexive muscle activation responding to the jerk loading is modeled by the torque actuator with a PID close loop feedback control. Considering that the test subject tries to keep the initial elbow joint angle, torque $\left(\mathrm{M}_{\mathrm{z}}\right)$ is activated to minimize the error which is the difference between the initial and current elbow joint angles. Meijer et al (2013), and Brolin et al (2015) presented successful applications of the active torque with PID control to their active human body models. Gain values for the PID control, i.e., Proportion, Integral, and Derivative terms determine the rates of torque generation. Faster torque generation with larger gain values stands for the recognition of jerk loading, i.e., "open eye" condition in the subject test. On the other hand, "closed eye" condition for unrecognized and thus more reflexive response that is modeled by smaller gain values. Comparison of hand displacements between subject test and simulation for four cases are shown in Fig. 5. The comparison of hand position at the maximum elbow extension between subject test and simulation is also shown in Fig. 6. 


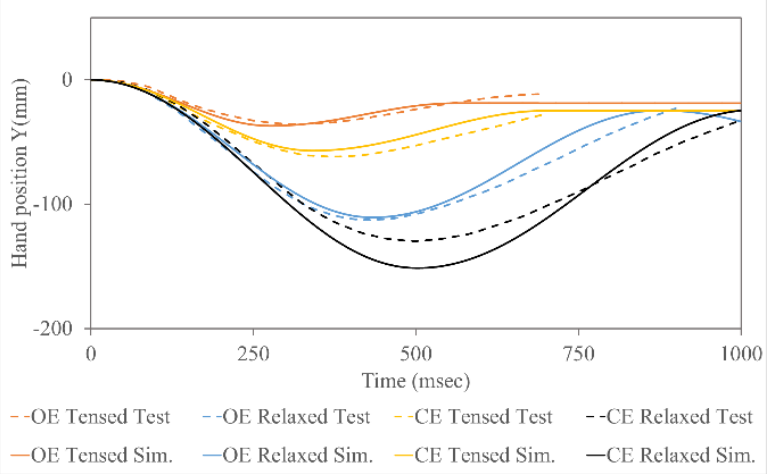

Figure 5 Comparison of hand displacements between subject test and simulation.
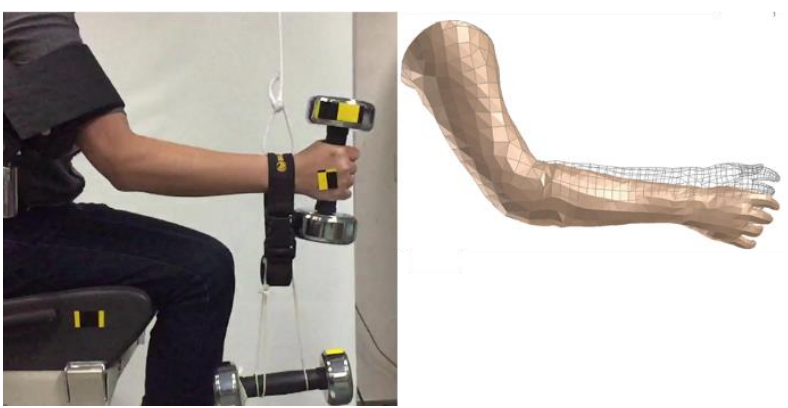

(a) Open Eye Tensed
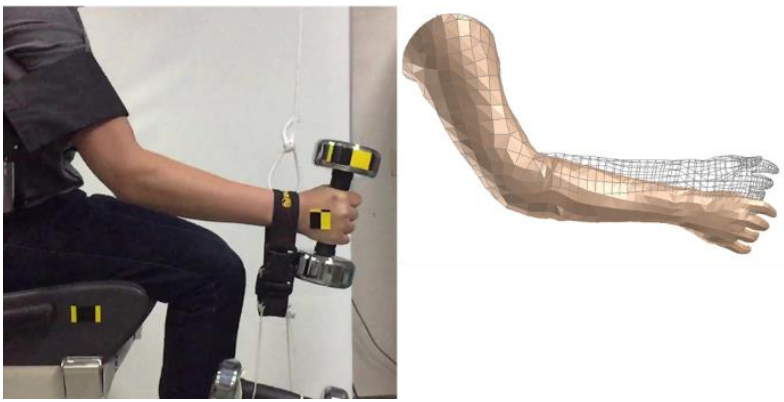

(b) Close Eye Tensed
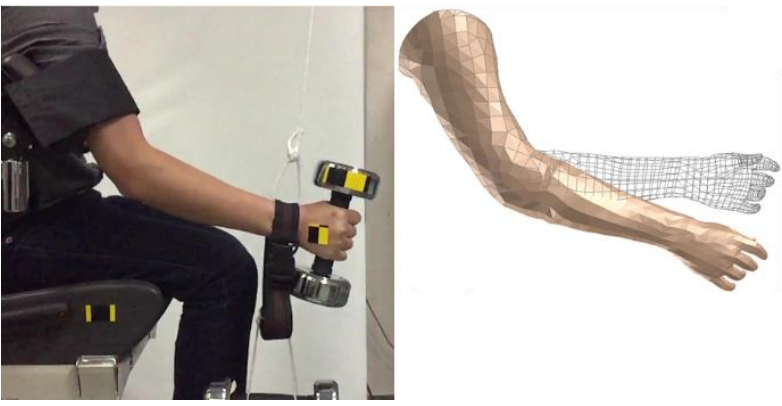

(c) Open Eye Relaxed
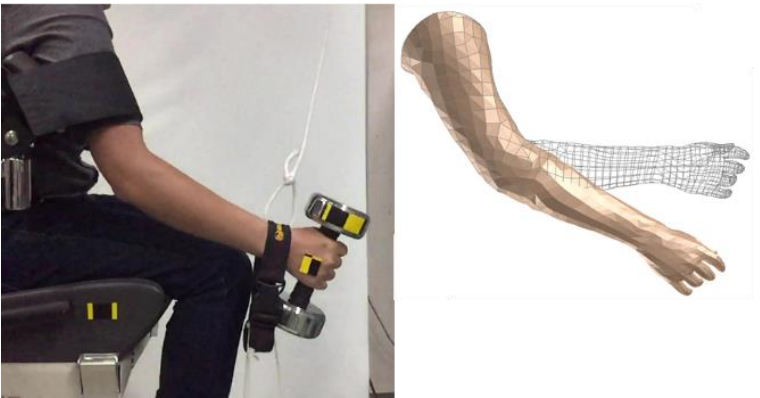

(d) Close Eye Relaxed
Figure 6. Comparison of hand position at the maximum elbow extension between subject test $\left(1^{\text {st }}\right.$ trial of Subject \#4) and simulation

\subsection{Hypothesis on the modeling of active elbow response}

Table 3 lists modeling parameters of active elbow joint for all four cases. The derivative term in PID close loop control turns out to be insensitive in this simulation of active response to the jerk loading and thus excluded. Those parameters were estimated by heuristic method (trial and error) and based on following hypotheses;

1. Muscle condition e.g., co-contraction (tensed condition) vs. single contraction (relaxed condition) is modeled by damping coefficient of $\mathrm{K}$-joint as shown in Fig. 7.

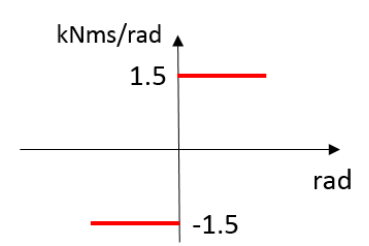

Co-contraction

(Tensed condition)

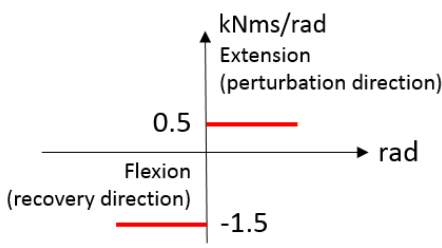

Single contraction (Relaxed condition)
Figure 7 Damping coefficients for different muscle tensing conditions

2. Stiffness of K-joint is dependent on inter subject variations, e.g., muscular structure, gender, etc.

- Muscular build (stronger) arm $\uparrow$, male $>$ female, and so on.

3. Recognition of perturbation (Open eye vs. closed eye) is controlled by gains of PID close loop control

- Relaxed condition (single contraction) has decreased gains by factor of 0.35 than tensed condition (cocontraction)

4. Muscle reflex latency (delay), $30 \mathrm{~ms}$ is given to the closed eye condition

Table 3 Modeling parameters and CORA score of active elbow joint model

\begin{tabular}{|c|c|c|c|c|}
\hline $\begin{array}{c}\text { Modeling } \\
\text { parameters }\end{array}$ & $\begin{array}{c}\text { Open eye } \\
\text { Tensed }\end{array}$ & $\begin{array}{c}\text { Close eye } \\
\text { Tensed }\end{array}$ & $\begin{array}{c}\text { Open eye } \\
\text { Relaxed }\end{array}$ & $\begin{array}{c}\text { Close eye } \\
\text { Relaxed }\end{array}$ \\
\hline $\begin{array}{c}\text { Damping C. } \\
\text { kNms/rad) }\end{array}$ & $-1.5 / 1.5$ & $-1.5 / 1.5$ & $-1.5 / 0.5$ & $-1.5 / 0.5$ \\
\hline $\begin{array}{c}\text { Stiffness } \\
(\mathrm{kNm} / \mathrm{rad})\end{array}$ & 0.1 & 0.1 & 0.1 & 0.1 \\
\hline $\begin{array}{c}\mathrm{K}_{\mathrm{p}} \\
(\mathrm{kNm} / \mathrm{rad})\end{array}$ & 80 & 50 & $80 * 0.35$ & $50 * 0.35$ \\
\hline $\begin{array}{c}\mathrm{K}_{\mathrm{i}} \\
(\mathrm{kNms} / \mathrm{rad})\end{array}$ & 0.015 & 0.015 & $0.015 * 0.35$ & $0.015 * 0.35$ \\
\hline $\begin{array}{c}\text { PID Control } \\
\text { Latency(ms) }\end{array}$ & 0 & 30 & 0 & 30 \\
\hline $\begin{array}{c}\text { CORA } \\
\text { Score* }\end{array}$ & $\mathbf{0 . 9 1 6}$ & $\mathbf{0 . 8 9 7}$ & $\mathbf{0 . 9 5 0}$ & $\mathbf{0 . 8 9 2}$ \\
\hline
\end{tabular}

* Calculated between test and simulation in Fig. 5

The correlation between test and simulation results for all four cases are qualitatively analyzed by CORA 
score as in Table 3. Open eye condition, i.e., recognition of jerk loading, shows better correlation slightly for both tensed and relaxed muscle conditions than closed eye condition. $(0.916,0.950>0.897,0.892)$

\section{Whole body modeling}

The same modeling scheme of active response at elbow joint is extensively applied to the whole body model. The version of multi-body model (c.f., deformable body model) consists of 15 rigid body segments and 14 articulated joints (Fig. 8). Each articulated joint has either 1 DOF (e.g., elbow, knee, etc.) or 3 DOF (e.g., shoulder, hip, spine, etc.) depending on its biomechanical characteristics. Same kind of passive kinematic joint element and active torque as in the active elbow joint model are implemented but its mechanical characteristics, e.g., the moment-angle curve and damping coefficient are dissimilar to each other. The errors to be removed by active torques at articulated joints are a composite function of joint angle changes at every body segments. Human driver voluntarily and/or reflexively braces to maintain upright sitting posture against various kinds of $\mathrm{G}$-forces during vehicle maneuvering such as emergency braking, lane change, cornering, etc. Validation of active human body model against the test data from open literature (Huber, 2015) is now in progress.

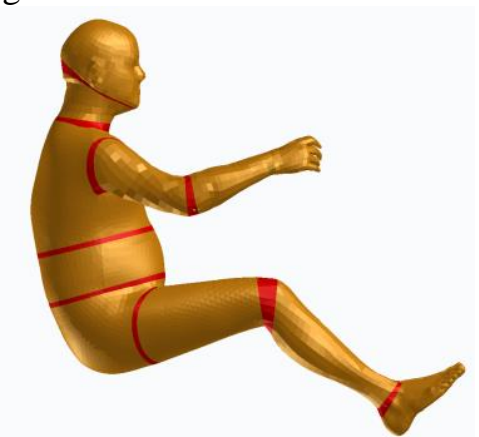

Figure 8 Whole body model with 15 rigid body segments and 14 articulated joint

\section{Discussion}

The SISO (Single-Input Single Output) problem with $1 \mathrm{D}$ active elbow joint model becomes MIMO (MultipleInput Multiple Output) problem with the whole body model. Human driver's muscle recruitment strategy of active response to brace against external perturbations belong to the quite complicated behavioral kinesiology. Also inter and intra subject variations make the active human body model as one of exciting challenges.

\section{References}

Karin Brolin, et al. Development of an Active 6 Year Old Child Human Body Model for Simulation of Emergency Events, IRCOBI 2015.
Huaining Cheng et al. (1996) The development of the GEBOD program, Biomedical Engineering Conference, Proceedings of the 1996 Fifteenth Southern

Hyung Yun Choi, et al, Experimental and numerical studies of muscular activations of bracing occupant, ESV 2005

Phillip Huber, et al, Passenger kinematics in braking, lane change and oblique driving maneuvers, IRCOBI 2015

Riske Meijer, et al. Modelling of Bracing in a Multi-Body Active Human Model, IRCOBI 2013. 